\title{
Review of Digital Twin applications in manufacturing
}

In the Industry 4.0 era, the Digital Twin (DT), virtual copies of the system that are able to interact with the physical counterparts in a bi-directional way, seem to be promising enablers to replicate production systems in real time and analyse them. A DT should be capable to guarantee well-defined services to support various activities such as monitoring, maintenance, management, optimization and safety. Through an analysis of the current picture of manufacturing and a literature review about the already existing DT environment, this paper identifies what is still missing in the implemented DT to be compliant to their description in literature. Particular focuses of this paper are the degree of integration of the proposed DT with the control of the physical system, in particular with the Manufacturing Execution Systems (MES) when the production system is based on the Automation Pyramid, and the services offered from these environments, comparing them to the reference ones.

This paper proposes also a practical implementation of a DT in a MES equipped assembly laboratory line of the School of Management of the Politecnico di Milano. The application has been created to pose the basis to overcome the missing implementation aspects found in literature. In such a way, the developed DT paves the way for future research to close the loop between the MES and the DT taking into consideration the number of services that a DT could offer in a single environment.

Keywords: Industry 4.0, Manufacturing, Digital Twin, simulation, MES, CPS, Cyber-Physical Systems

\section{Introduction}

With the new paradigm of Industry 4.0, manufacturing evolves to include smart objects [1], basing on the concept of Cyber-Physical Systems (CPS), defined as "systems of collaborating computational entities which are in intensive connection with the surrounding physical world and its on-going processes, providing and using, at the same time, data-accessing and data-processing services available on the internet” [2]. These new autonomous systems are capable to elaborate and communicate data and to build a copy of real processes in a digital environment in real-time [3].

The main enabling technologies of the Industry 4.0 that characterise CPSs (such as Big Data and Cloud computing as support systems to read big sets of data from the field, store and analyse them [4,5] and Internet of Things (IoT) to remain connected and extract data) are also the basis for a new simulation approach, which leverages on the pervasive connectivity in production systems to offer a 
real-time synchronization with the field. This new simulation approach is generally referring to the elaboration of Digital Twins (DT) [6]. Although much literature was produced on the topic, a general definition and an agreement over its features and scopes has not been reached yet. Considering its close link with production management issues, it becomes highly valuable to understand the features of the applications of DT and to compare them with what is suggested by literature. This paper aims at exploring the existent literature about the practical applications of DT, identifying the gaps between the theoretical features of the DT and the applications. Then, this paper proposes an application in a laboratory environment that poses the basis to overcome the found gaps.

To this aim, the next part of the introduction this paper is structured as follows: firstly, the automation pyramid structure, the most commonly used structure in industrial field, is illustrated in section 1.1. Then, in section 1.2 the innovations in this field are showed highlighting the role of the mentioned CPSs and the related Digital Twin (DT). Finally, in section 1.3 this paper will condense the main points for a reflection on the current scenario, needed to better illustrate the objectives of the paper.

\subsection{Relation to the Automation Pyramid}

Each industrial automated process is commonly based on the Automation Pyramid, a centralized structure composed of five layers, as in Figure 1 [8]. On the left of the figure lies the automation pyramid (https://visaya.solutions/video/old-new-automation-pyramid/), on the right the corresponding production management level [9]. The figure also shows the Manufacturing Execution System (MES) functions listed by the Manufacturing Execution System Association [10].

(i) The three lower layers (0 - 1 - 2) are summarized as the control layer, including: electrical engineering layer, Programmable Logic Controllers (PLCs) / Distributed Control 
Systems (DCS) and Scada/HMI layers,

(ii) the middle layer (3) is the Manufacturing Execution System (MES) that guides the process, and

(iii) finally on top there is the Enterprise Resource Planning (ERP) layer (4) aimed at integrating organizational functions for better customer support and planning [11].

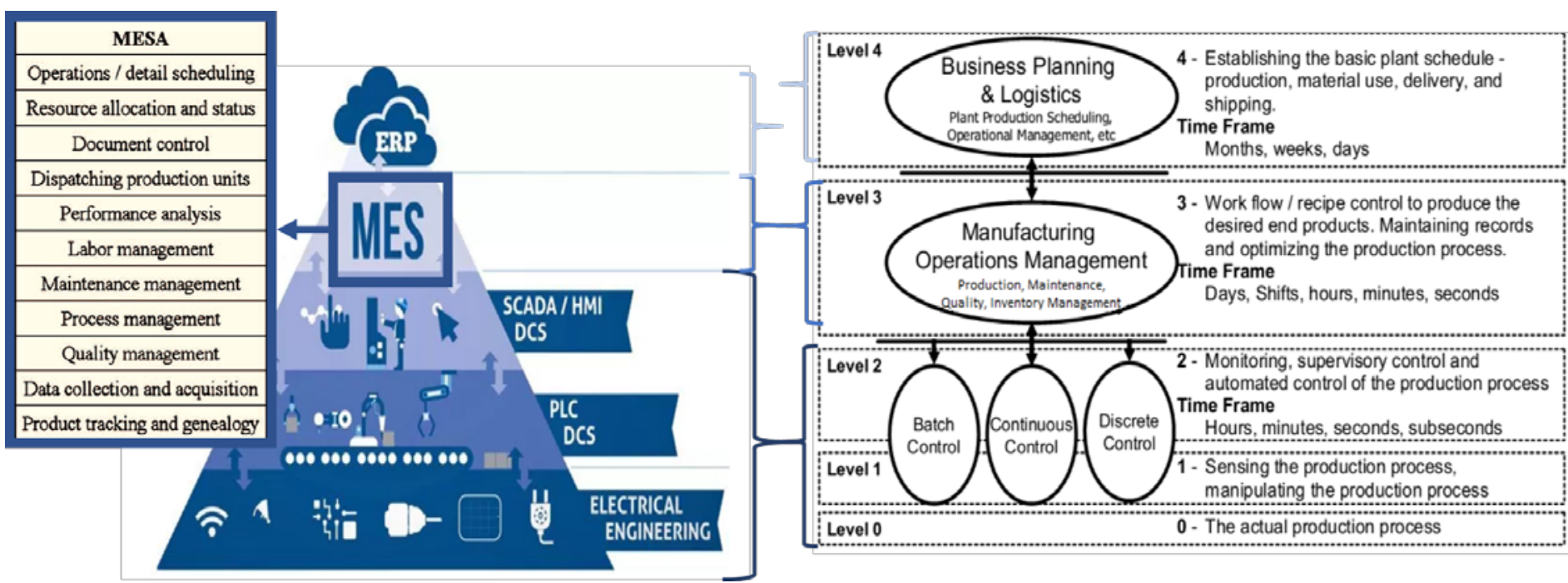

Figure 1. The automation pyramid, the corresponding production management level $[9,12]$.

In this structure, the MES guides the manufacturing process, monitoring all the steps of a product in the production system in a centralized way. Among the MESA-identified functions, some are directly linked to the production process (such as scheduling and quality control), while others (such as resource management and traceability) are best described as cross functions, not strictly related to the production process but supporting a higher point of view. Thus, all MES functions are not on the same level, as suggested by De Ugarte [13].

\subsection{Digital Twin in CPS-based systems}

Introducing the new technologies mentioned above, manufacturing industries future vision is often depicted in literature as based on CPSs [14] or on Cyber Physical Production Systems (CPPS) [15] and on the associated concept of the DT. 
CPSs represent a promising future within the Industry 4.0 context and offer to enforce the flexibility of the automation pyramid-based manufacturing systems [16], showed in section 1.1. CPSs, by definition, have both a cyber and physical nature. In the cyber part, they will be suitable to host computations, that cover data analysis and on-board simulations. The simulation may be:

- synchronized with the field (physical part of the CPS) but is limited to the boundaries of the CPS itself (e.g. a single workstation);

- a higher-level simulation, that is hosted outside the CPS system itself. This simulation may in this way replicate both single workstations and the whole production system behaviours, as data are collected from the single CPSs.

The latter type of simulation, when synchronized with the field, complies in all aspects with the definition of DT given by [17] and reported in [6,18]: “The DT consists of a virtual representation of a production system that is able to run on different simulation disciplines that is characterized by the synchronization between the virtual and real system, thanks to sensed data and connected smart devices, mathematical models and real time data elaboration. The topical role within Industry 4.0 manufacturing systems is to exploit these features to forecast and optimize the behaviour of the production system at each life cycle phase in real time”. In this vision, the future of manufacturing is characterized by entities, CPSs or CPPSs, able to collect data directly from the field and to replicate the physical production system in the cyber world through various digital models that compose a DT; i.e. these models open the way to a real-time synchronized simulation of the physical equipment [6]. The digital models also encompass a proper data modelling to allow for interoperability of various models and tools, to offer a common vocabulary and to provide to the system knowledge about the information the digital models elaborate: all of this is well supported by semantic models, such as ontologies [19,20].

As said, the DT is related to the CPS and is seen as its digital counterpart. The concept of DT is still evolving and its definition has matured over the years as Negri reports in [6]. The DT was initially 
employed by the NASA to replicate the life of the air vehicles. DT were used for health analyses i.e. crack propagation [7], or for improving maintenance activity and planning. Then, it was also introduced to digitally mirroring the life of the physical entities and to support decision making through engineering and statistical analyses; in this wider perspective it started to be used also in other fields such as industrial engineering [17]. In its essence DT can be considered as an environment that can support different types of simulation or is considered as a simulation itself, which is synchronized with the field in a near to real-time fashion.

An interesting interpretation is given by Kritzinger, that proposes in [18] a review of the DT literature depending on the interactions between the physical object and the relative virtual object. In this sense, a proper DT is the one where the virtual object exchange data flows with the physical one in both directions (physical-to-virtual and virtual-to-physical directions). This means that the virtual object can eventually act on the control system of the real one. This DT interpretation is depicted in Figure 2 together with the other two categories mentioned by [18]. (i) The Digital Model does not entail any interactions between the physical and virtual objects. (ii) The Digital Shadow, where only the physical object sends data and updates the virtual one. It includes the Digital Model, the data acquisition protocols and the simulation software to simulate the digital model. In this case, the interaction between cyber models and physical objects is monodirectional. (iii) As stated by Kritzinger [18], and illustrated in the context given by this paper in Figure 2, the Digital Shadow can become a full Digital Twin only when the dotted line connecting data coming from the Digital Shadow (the virtual twin) with the real system is realized, therefore when the data flow is bidirectional from the real system to the DT and vice versa. 


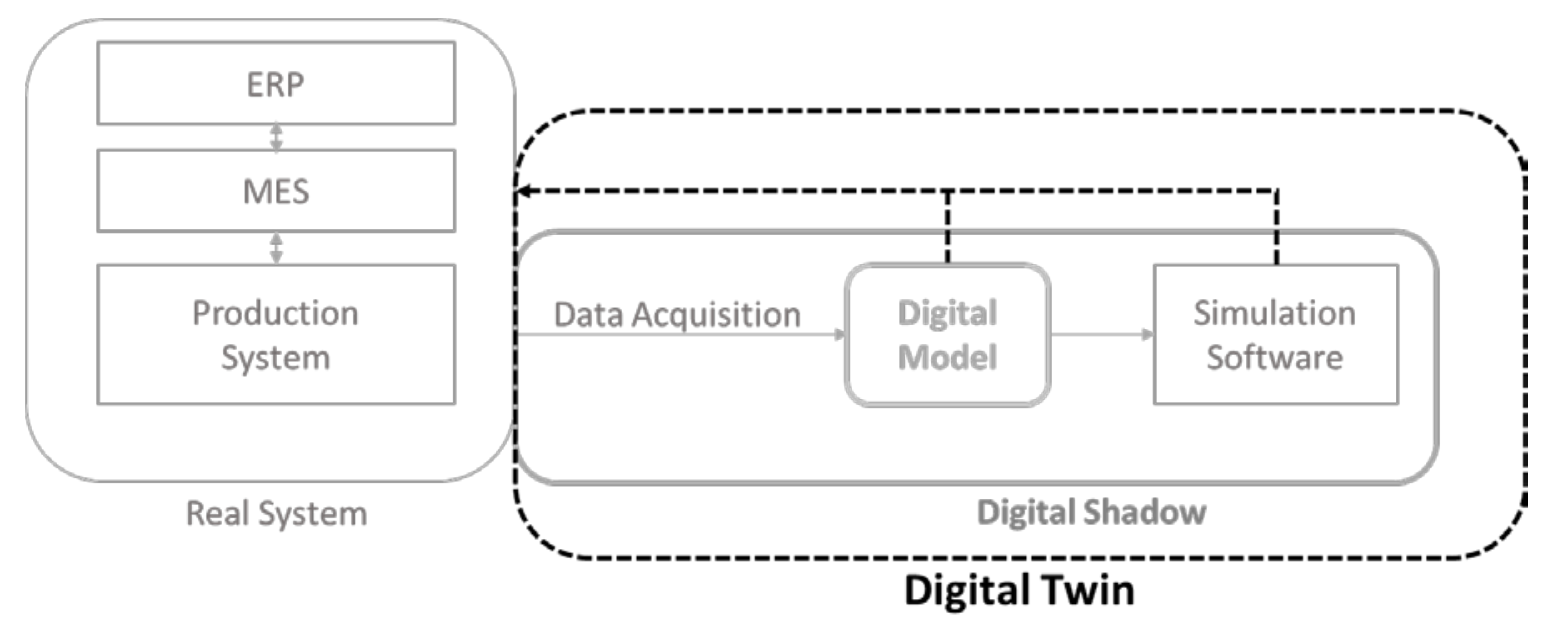

Figure 2. Digital Twin (DT) schema

The DT is often used to offer specific analyses, related to the considered system and to its lifecycle, according to the services that a DT may offer, as listed by Tao et al [21]. As we will recall later, not all services are needed for all systems, but having them at disposal in one DT environment may be useful for industrial decision makers that do not need to carry out different analyses in separate digital environments.

Considering the nine services listed by [21], they can be grouped in these categories:

- Real-time state monitoring, used to update the virtual twin in real time;

- Energy consumption analysis;

- Product failure analysis and prediction, and product maintenance strategy, that have in common the analysis of the real-time state data and historical data to predict a fault and construct a maintenance strategy;

- Intelligent optimization and update; this service is based on the analysis of the user's operation habits and product behaviours data to improve the product and/or the production process;

- Behaviour analysis and user operation guide, used to obtain the operations done from the users and/or giving them some user guidance to visualize the system updates with a userfriendly HMI (Human-Machine Interface); 
- Product virtual maintenance and product virtual operations; given a 3D environment or software, these services elaborate and show the operations or the maintenance strategy to the user.

The challenge in offering these services in a single environment leads to the fact that some of them need a 3D graphic interface and others only analyse data without requiring any graphics. Also noticeable is that some of them (e.g. "Intelligent optimization and update") are based on this mutual exchange of information between the real and virtual object, the DT must offer specific services, that recalls again the concept introduced by Kritzinger [18].

\subsection{The current scenario}

To sum up, the adoption of a DT must ensure that it is connected to the physical twin. In case the physical system it mirrors has the characteristics to be considered a CPS, this will make the DT implementation easier because its synchronization passes through a direct field connection hosted by the CPS itself. Also, the DT does not have a unique definition or a unique reference model yet, but Figure 2 provides the reference concept of DT for the purpose of this work.

In practice, research on CPS and DT is still ongoing, and in most cases manufacturing systems at companies are equipped with traditional machinery which is hierarchically based on the automation pyramid: the production is planned in the long run by the company information systems (such as ERP), and the sequence of operations is controlled by the MES of the systems (referring to the levels of the automation pyramid of Figure 1). In these cases, it is more difficult to introduce an implementation of DT in the systems and to integrate it with an existent MES-based equipment.

This paper wants to investigate the DT applications present in literature, to understand how the DT is used practically on real systems and what are the services offered. Particular attention will be given to the improvement that can be made to boost the usage of a DT inside the already existent systems, given the importance of the additional information and analysis provided by this tool.

The structure of this paper is the following: in section 2 the objective and approach of the work are 
better outlined; section 3 reports the literature review about the DT applications; in section 4 the creation of a DT in a laboratory environment and the related results are described; section 5 discusses the created DT environment; section 6 proposes hints for future work and final remarks.

\section{Objective and approach}

As already said, research about DT is ongoing as a promising topic for decision making in various fields, among which the manufacturing field is one of the most relevant [22]. The present paper aims at proposing an overall vision on the DT application for production systems. The main objective is to identify the discrepancies between literature and implementations of DT, starting from a literature review on the DT applications. The paper also aims at proposing a DT environment in laboratory where this topic can be further studied, posing the basis to overcome the discrepancies found.

More in detail, the literature review investigates the practical DT applications in an industrial or laboratorial environment, relating them to the definition of DT given in literature and to the services proposed by [21]. This review is carried out in line with the definition of DT in the classification done by [18].

\section{Review of Digital Twin applications in literature}

As mentioned, researchers and practitioners still do not agree on a unique definition of DT. A lot has been said about its role in the Industry 4.0 paradigm, however, only few practical examples are reported in scientific papers that do not show the same technological features and functionalities. It is therefore of paramount importance to investigate previous research works about DT application in production environments. Scopus was used as research literature database, considering the papers in English language in the last 5 years (starting from 2015 to 2019). The reason for the choice of this time span is that DT in production systems in the Industry 4.0 meaning were first proposed in 2015, as shown by [6]. As reported before, this paper wants to investigate the practical implementations of the DT of production systems in industry or laboratory environments, 
understanding the principal application purpose of the DTs created, which services are offered among the ones described by [21] and how the architecture for data acquisition and simulation was built (including dataset acquisition and protocol, model and used software). For this reason, the literature research was executed on Scopus with the logical expression of keywords: (“digital twin”) AND (“manufacturing” OR “production” OR “operations”), updated until April 2019.

Regarding the research, the total number of result articles were 309, among which several articles had to be excluded as they were not useful for the purposes of the analysis (point 1 in the “Objectives and approach” section) for the following reasons:

- Some of them did not explain the development of the DT and the architecture to support it;

- Others were only theoretical articles, dealing with DT or more in general about Industry 4.0 without showing any practical application;

- Some articles did not actually report applications in the manufacturing domain;

- Some others were not downloadable.

As a result, 52 articles were included in the analysis, all reporting DT applications in industrial or laboratory manufacturing environments. Table 1 reports the results of the analysis of the articles. 


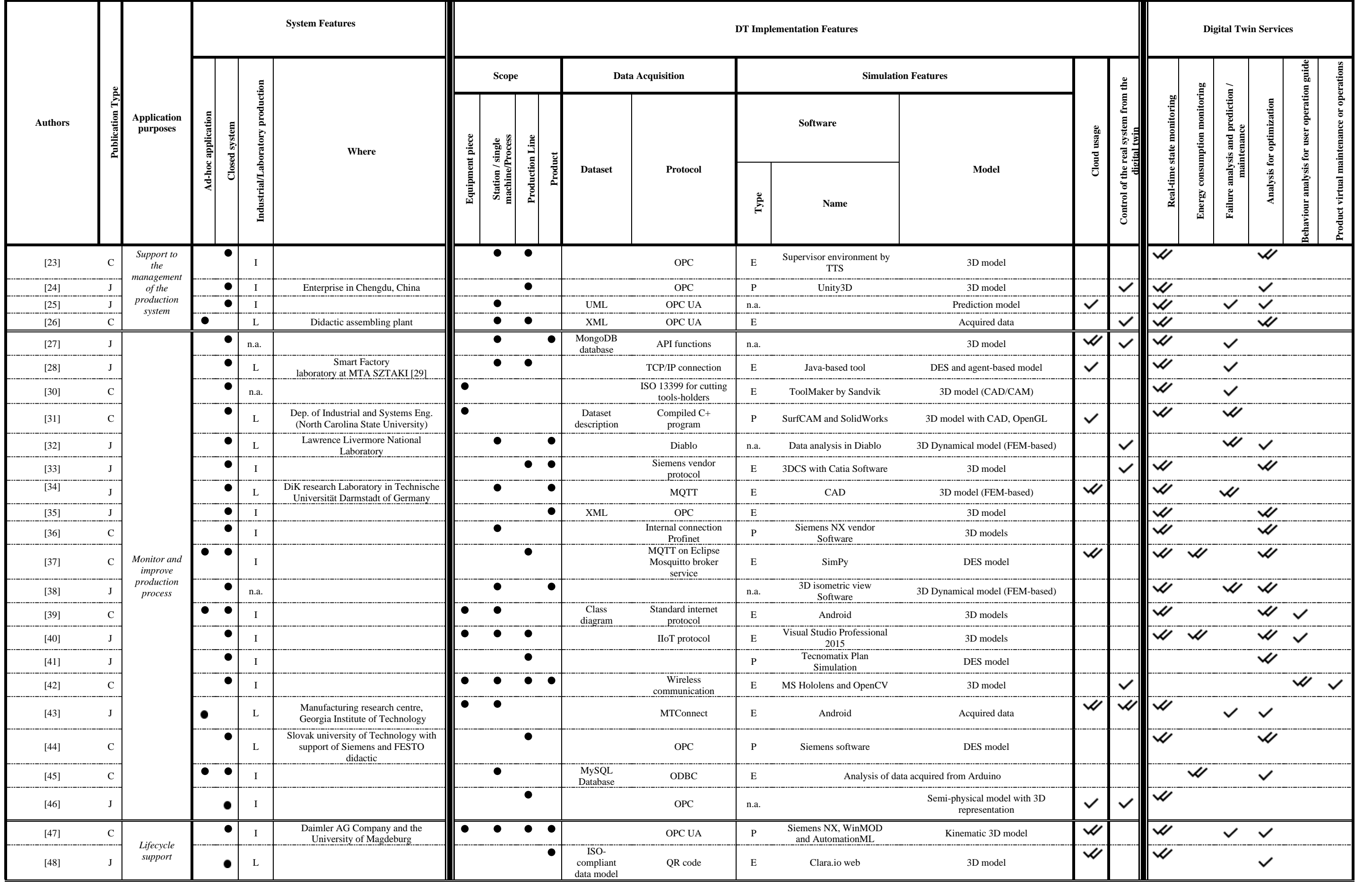




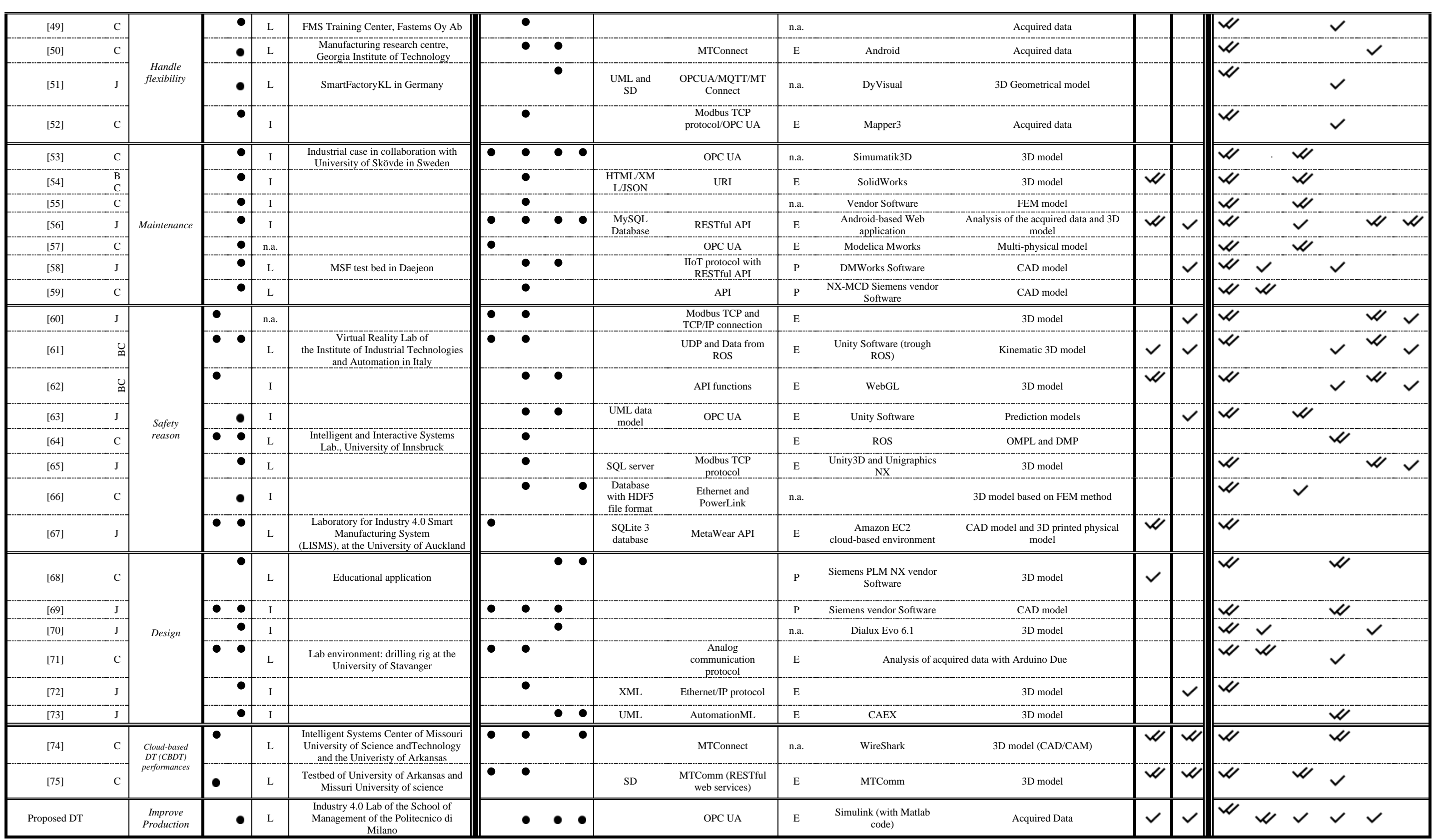


Table 1. Literature review about the DT applications (Legend: $w$ means that the characteristic has been considered and implemented in the DT application; $\vee$ means that ithas been considered but not implemented). 
The table reports several articles - one in each row - identifying the name and year of publication (in the first column) and the type of publication, whether it is a Journal publication (J), a conference contribution (C) or a book chapter (BC) (in the second column). It is possible to notice that most of the papers (29) were published during the year 2018. Twelve were published in 2019 (until April). The rest of papers (11) included in Table 1 are from 2017. This suggests that the research on this subject is still ongoing and is growing in the recent years. Among the articles selected, 24 were presented to conferences and 25 published in journals, three are extracted from three different books, demonstrating the high scientific importance of the treated subject. In Table 1, the articles are clustered following the "Application purpose" for the development of the described manufacturing DT. This column shows that the DT can be constructed for a variety of different purposes:

1) The DT can be used to "Support the production system management" with the construction of a DT called “installer base management system” that assists in data structuring and management of machines [25], identifying the design for automated flow-shop manufacturing systems [24], supporting the supply chain management through data monitoring [23,26];

2) The DT can help to "Monitor and improve the production process", sometimes optimizing it: this is done with the creation of a system architecture for the virtualization of manufacturing machines (VMM) [27], integrating multiple dynamic simulations [28], monitoring a machine tool [30,31], monitoring product parameters during production [35], a single process $[34,36,44,45]$, an assembly process $[33,41]$ or a production line $[42,43,46]$. The proposed improvements are reached by reducing the energy consumption [37,40], increasing the productivity performances [39], or limiting geometry faults in the additive manufacturing processes [32,38]; 
3) The DT can also be used to "Support the lifecycle" of a machine process [47], like in material recovery [76] [48].

4) The DT application is also used to "Handle the flexibility" of the production systems [4952];

5) Other articles introduce the DT for "Maintenance" purposes [48,54-59], such as reconditioning of old machines [53];

6) DT was also considered helpful for "Safety reasons" in human-robot interaction [61,63,64], in monitoring of a smart wearable equipment [67], or in the manufacturing process of a produced part [66], within a virtual reality (VR) visualization platform creation [65] or with a HMI visualization environment [60].

7) In some cases, the DT is used for the "Design" of a machine [68,72], of a laboratory environment [71], of a product or production environment $[69,73]$ or for the redesign of the working environment [70].

8) Finally, the DT was also used to evaluate the "Performances of a cloud-based DT", that are mainly aimed at verifying the speed with which the data are updated in a cloud environment $[74,75]$

The remaining columns of Table 1 are commented in the next sections.

\subsection{System features}

The "System Features" columns describe the system type taken into consideration in each article. Accordingly, the production systems can be grouped in three categories, that are listed below. Each article is marked with a black circle according to the type of production system:

- The "Ad-hoc system" is a production system which is set up for the purpose of the DT 
research reported in the article. It is therefore relying not on closed proprietary systems, but on open protocols and programmable boards. In Table 1 seven articles developed "ad-hoc" systems to build DT: for the management of a didactic production line [26], to construct a MES in Android systems [43], to build user guides for safety reason [60,62], and to evaluate the performances of a cloud-based DT [74,75];

- The "Closed system" is a closed and proprietary production system, in industrial environments and sometimes also in laboratories. The considered system is synchronized with DT simulation directly without the use of additional sensors or other external devices to build the DT application since the DT simulation usually runs on simulators that are native or from the same vendor of the production system;

- The "Hybrid systems", the systems in Table 1 where both the boxes "ad-hoc" and "closed system" are checked. These systems are closed proprietary ones but some "ad-hoc" elements are included to realize the DT environment, i.e. additional sensors or programmable boards. In some cases the production system is equipped with additional sensors to perform different analyses [37,39,45,67], or for the design of a single process [71]. In other cases, the system is considered with the addition of cameras: in particular, in one case the system is a production robot that will be in the future controlled from data coming from the cameras through its DT [61], in another case is used for remote training of a robot [64] and in the last case is used to evaluate the design of a process line [69].

- The "Industrial/Laboratory production" column reports whether the production system on which the applications were developed are industrial (I), laboratory (L) or if there are no information about it (n.a.).

- Additional details on the type of laboratory and/or its location are given in the "where" column. 


\subsection{DT Implementation Features}

The columns under "DT Implementation Features" in Table 1 reports the architectures used to support the DT.

- First, the analysis highlights the "Scope" of the DT, i.e. the level of detail of the system replicated in the DT. It can be at different levels of details: equipment piece, workstation or production system level or applied only at the product level. There are aspects that can be faced from each level of detail, i.e. [42,47,53,56]; in other cases DT can be developed contemporarily for two or three different levels of detail, as in $[23,40]$; but in most of the cases DT are usually developed on a single scope level, i.e. [24,30,31]. In most cases, the scope is strictly related to the application purpose, e.g. for the purpose "Support to the management of the production system” both the machine/process of the production line level of details are investigated; the equipment piece and the product scopes are not used, as they would not give any additional information for that purpose. The same concept can be extended also for the application purpose "Handle flexibility", related only to the machine/process or to the production line. The other "Application purposes" are instead applicable to all the scopes in Table 1.

- The "Data acquisition" section shows details about the "Dataset" and "Protocol" to acquire data from the production system.

o The former checks if the analysed contributions explicitly mentioned the formalism of the acquired dataset and the acquisition protocol. The dataset description is showed only in nineteen articles: in four cases a description is done using Unified Modeling Language (UML) [25,51,63,73], in one case only with the Sequence Diagram (SD) [75]. In other cases, the description is less detailed and shows only a dataset description with machine data [31] a class diagram description of the data [39], an XML format of the data $[26,35,54,72]$. In the rest of the cases different 
environment are used to describe the dataset, like HDF5 File [66], MongoDB [27], SQL Database[45,56,65,67] or ISO-compliant data model [48].

o The acquisition of the data is always performed with the use of a protocol. From Table 1, it appears that different alternatives are possible but the Open Platform Communications Unified Architecture OPC UA and Transmission Control Protocol/Internet Protocol (TCP/IP) connection seem to be the most used.

- The "Simulation Features" considered in Table 1 are strictly related to the considered type of system. If a system is closed and proprietary (as defined in "System Feature" section), the used software of the DT is most of the times the system vendor one. This section reports the "Software" used to describe the "Type" of the simulation environment and the "Name" when available, of the software tool used, finally what type of simulation "Model" was developed.

o The software can be of different "Types": external (E) and adapted to the system, therefore opening the possibility to further re-adaptations to a variety of other systems. The software can also be proprietary (P) and specific for the system considered. If there is no mention to the used software, "n.a." is written in this column.

o From the "Name" column, it can be noted that there is not a prevalence of one environment with respect to the others because the environment is directly related to what information must be extracted. This is also related to the model used to analyse the data extracted.

o Regarding the "Model", the articles selected in Table 1 can be divided into different categories. Five articles use a 3D dynamical Finite Element Method (FEM)-based model $[32,34,38,55,66]$ and one that uses a multi physical model to represent different physical aspects [57]. In two cases is used a prediction model [25,63], in other cases the representation of the production system is done with a Discrete Event System (DES) model [28,37,41,44]. Eight articles did not use any model, but they 
extract information from the system experimentally, analysing the acquired data $[26,43,45,49,50,52,56,71]$. The most used model is the 3D kinematical model, used by the remaining articles in Table 1.

- Another feature to consider in the "DT implementation Features" is the "Cloud Usage”. For this aspect, the articles considered can be divided into two different categories: (i) those that effectively use a cloud and build a cloud-based DT application, marked in the table as $w$ $[27,34,37,43,47,48,54,56,62,67,74-76]$, (ii) those that construct the DT application in a virtual space with the use of a software highlighting the possibility to build it also in a cloud-based environment, marked in the table as $\checkmark[25,28,31,46,61,68]$. If the cell is not checked it means that no mention to the cloud has been made in the article.

- The last important "DT Implementation Feature", "Control of the real system from the digital copy", responds to the question "Is the real system controllable from the DT?” and is related to the services offered by the DT environments. Tao gives indications on what are the services that a DT must offer, which are all requiring the DT to be connected to the real system [21]. For this reason, this feature highlights if the real system is effectively controlled by the digital counterpart (marked as $w$ ) or at least could be controlled by it in future works (marked as $\checkmark$ ). If the cell is not checked it means that no mention to the possibility of controlling the physical system through the DT has been made in the article.

\subsection{Digital Twin Services}

The analysis also considered "Digital Twin services” offered by the DT environments taking as reference the services by Tao [21]. It is possible to classify the analysed papers in two groups:

1) DT that offer services connected to state monitoring, energy consumption, maintenance support and data analysis for optimization (in Table 1 these four services are the ones on the left); 
2) DT whose main services are visualization of the behaviour of the system or offering user guidelines, as an example through user-friendly HMI [56,60], or through virtual reality 3D systems in a software [42,61,65], or with the use of Smart Glasses [62].

Some DT applications in literature do not comply with one of the two mentioned groups, as they are developed specifically to offer one single service, such as only for system monitoring $([67,72])$, or for performance optimization ([41,64,73]).

Also, there are papers that mainly belong to the first group but also offer services of the second group $([39,40,50,70])$, and vice versa $([56,61,62])$.

The services offered can be analysed also in relation to the "Application purpose” that they want to fulfil with the DT construction. In some case the application purpose can be realized with different services, i.e. in case of "Monitoring and improve production process”, "Handle flexibility”, "Safety reason" and "Design" the improvement performed can be of different nature and can be realized with each of the services listed in Table 1. (i) In case of "Monitoring and improve production process” rarely the services "Energy consumption monitoring”, "Behaviour analysis for user operation guide” and “Product virtual maintenance operation” are offered; (ii) for "Safety reason”, the "Energy consumption monitoring" is never used, and rarely "Failure analysis and prediction/maintenance”; (iii) for “Handle flexibility” both "Energy consumption monitoring” and "Failure analysis and prediction/maintenance" are not considered; (iv) for "Design" purpose, "Failure analysis and prediction/maintenance" and "Product virtual maintenance operation" are not offered. (v) For the purposes “Support to the management of the production system” and "Lifecycle support” only the first four services in Table 1 can be useful, and it is worthy to note that for these types of purpose the "Energy consumption monitoring" is never used. (vi) In most cases, the purpose "Maintenance" is accomplished with the service "Real-time state monitoring" and the strictly related service "Failure analysis and prediction/maintenance”, rarely the other services are used also if they can be really useful, i.e. it could be helpful to know the profile of the energy through the "Energy consumption monitoring”. (vii) For “Cloud-based DT (CBDT) performances” 
the services offered are monitoring and the optimization, in the sense in this case of optimization of the connection established. In this case, providing the other services does not add real value for the CBDT creation. Nevertheless, providing all the services in the same DT application means to provide all the application purposes in a single environment, using each service for different purposes. From Table 1, it can be seen that no DT applications in literature give all the services proposed by [21], but at most two or three of them contemporarily.

\subsection{Overall findings of the Review}

To sum up, the DT can be applied at different scope levels, and in each case the required structure to build it seems to be the same. The schema identified is showed in Figure 2. The Real System is represented with the layers of the mentioned automation pyramid (Figure 2), the construction of the Digital Shadow starts from the data acquisition in a virtual environment, that can be done with different protocols. Successively, the acquired data are analysed and processed with the use of digital models that open the way to representing the real system in the chosen virtual environment with a (simulation) software. These first steps are needed to give the final shape to the Digital Shadow. As it can be seen in Figure 2, when the Digital Shadow is bidirectionally connected to the main controller of the real system (that is the MES in the case considered in Figure 2) for decision making, the overall system becomes a proper DT.

\section{First gap - DT integration with control system}

The majority of the proposed DT applications do not mention the connection of the DT environment to the control system of the physical equipment. The only articles that actually perform a control of the real system (i.e. in Table 1, column "Control of the real system from the digital twin”) are “ad-hoc systems” [43,74,75], meaning that they used open protocols and programmable boards. The reason could lie in the fact that "ad-hoc" systems do not have a centralized structure 
that controls the production/process in a MES-like way and because of this they can be controlled from the DT.

\section{Second gap - Offered DT services}

Only a limited set of services are offered in one single DT application, according to the classification made by Tao [21]. As said before, the scope is in most cases strictly related to the application purpose; rarely a DT of an equipment tool is scalable to the production line level. A comprehensive application of a DT of production systems that offers all services is not proposed among the ones in Table 1. Besides, it is a major challenge to offer a higher number of services in the same environment, because, certain services need a graphical representation and others just elaborate data without the use of graphics. Having all the services offered on the same environment can be useful in case of complex decision making, that needs different aspects analysed at the same time. The majority of proposed applications cannot fully offer the services from Table 1 by [21], and this places the related DT in the area of "Digital Shadows", that do not reach the full DT potentials [18]. In fact, without the bidirectional interchange of information between DT and its physical counterpart, there is no possibility to offer some services (e.g. optimization of the processes).

\section{Digital Twin creation}

This section illustrates the development of a DT that poses the basis to overcome the gaps found in the previous section. The proposed DT is built with the purpose of energy consumption monitoring, inside the Industry 4.0 Laboratory (I4.0 Lab) of the School of Management of Politecnico di Milano [77].

\subsection{The production line}

The production line installed in the laboratory, showed in Figure 3, was designed to assemble a 
prototypical mobile phone, with the following assembly steps (form 1 to 7 in Figure 3): the “Manual Station” (1), loading/unloading station where the production starts, the "Front Cover Station" (2) where the Front Cover is positioned on the pallet, the "Drilling Station" (3) where the cover is drilled, the "Robotic Cell" (4) where the Printed Circuit Board (PCB) and the fuses are placed inside the front cover, the "Camera Station" (5) controls that the pieces inside the front cover are well positioned, the "Back Cover Station" (6) places the back cover on the front cover, the "Press Station" (7) presses the two covers to close the piece. Finally the finished assembled part returns to the "Manual Station" (1) where the operator unloads it. Number 8 in Figure 3 is the bridge that can switch the production flow either to the robotic cell or to the camera station, according to the single mobile phone routing.

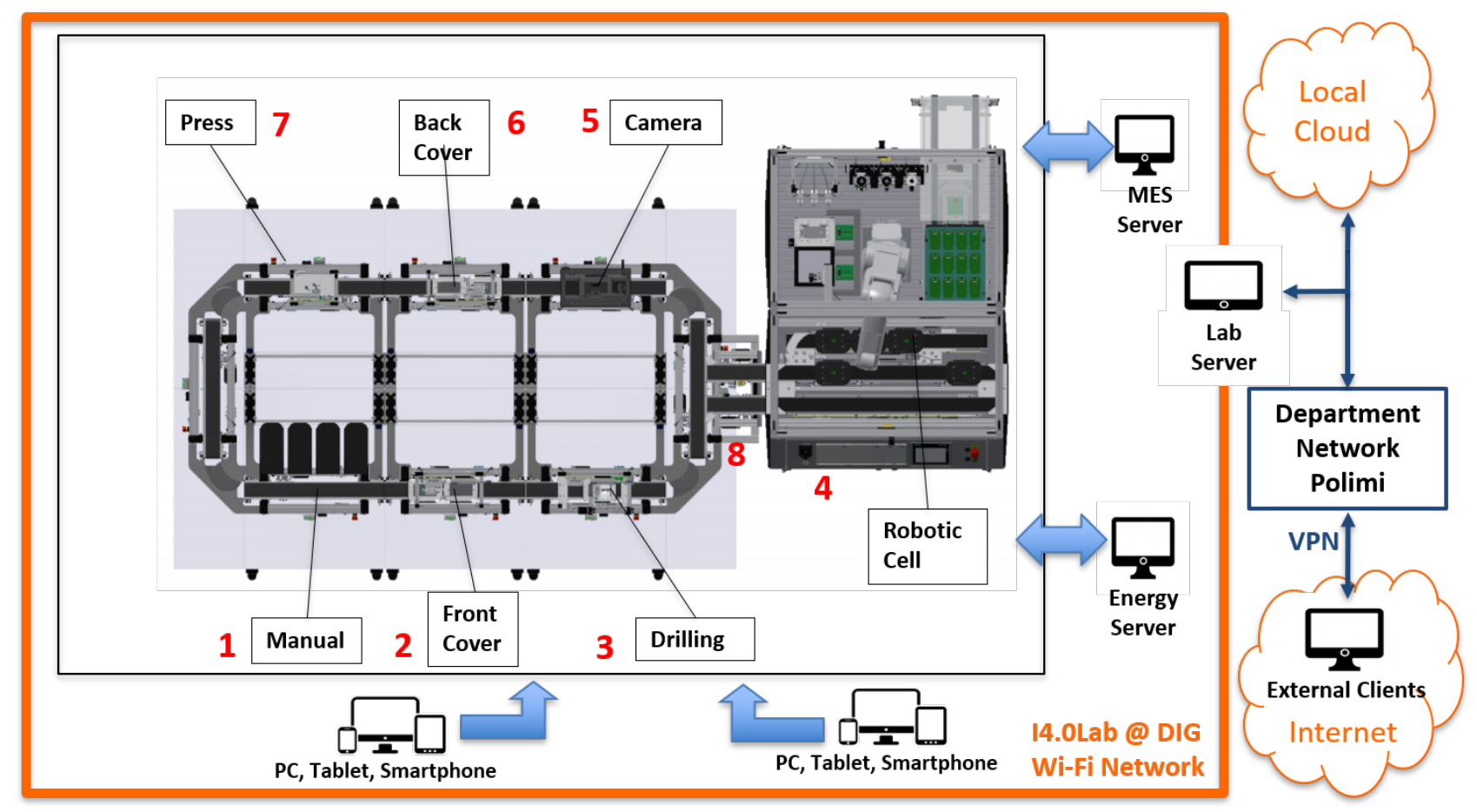

Figure 3. Industry 4.0 Laboratory production line

All the stations inside the Laboratory are equipped with: Band, PLC, Application module and HMI. There is no buffer, and the automated conveyor is the only handling system to move the products from station to station. In this system, a certain number of carriers moves around the line and are 
stopped in each station to perform assembly operations and in the meanwhile to read/write the information about the products using the Radio-Frequency IDentification (RFID) technology.

As showed in Figure 3, the entire production line in the laboratory is connected to two computers. The first one is equipped with the MES system from which the production orders are launched, and the production itself is controlled. The other computer hosts an application that monitors the energetic behaviours (i.e. electric current, power ...). All the stations of the line and the computers are connected to the laboratory network and have their unique IP address.

\subsection{Data Acquisition}

The I4.0 Lab is equipped with OPC UA, a machine to machine (M2M) communication, that has been used for Data Acquisition for the DT. This is the most recent OPC specification generated by the OPC Foundation that intends to unify all the existing OPC technologies. This type of protocol allows software tools to establish a unified architecture with industrial equipment and systems for data collection and control and is also already used for industrial automation, as appears in the literature review section (3.2).

OPC UA has also its own defined security mechanism, thus an extra encryption layer implementation is not needed. Further details and more description of design aspects of OPC UA for industrial application can be found in [78].

Matlab and Simulink are used for the modelling and for the Data acquisition inside the laboratory, following the indications by [79]. Matlab is a computing environment developed by MathWorks (https://it.mathworks.com/). Simulink, always developed by MathWorks, is the correspondent environment for dynamical simulations. They use the client/server implementations integrated in the OPC Toolbox ${ }^{\mathrm{TM}}$. 
This toolbox allows the user to work with data from live servers or historical data and conforms to the OPC UA standard required to exchange data to or from the servers (https://it.mathworks.com/products/opc.html?s_tid=srchtitle).

Since all the modules in the I4.0 Lab are equipped with PLCs that use OPC UA protocol, it is possible to create a connection in real time with the facilities by means of the OPC UA Toolbox. Figure 5 shows the OPC UA client/server connection scheme, where multiple clients can be created at the same time to query the servers.

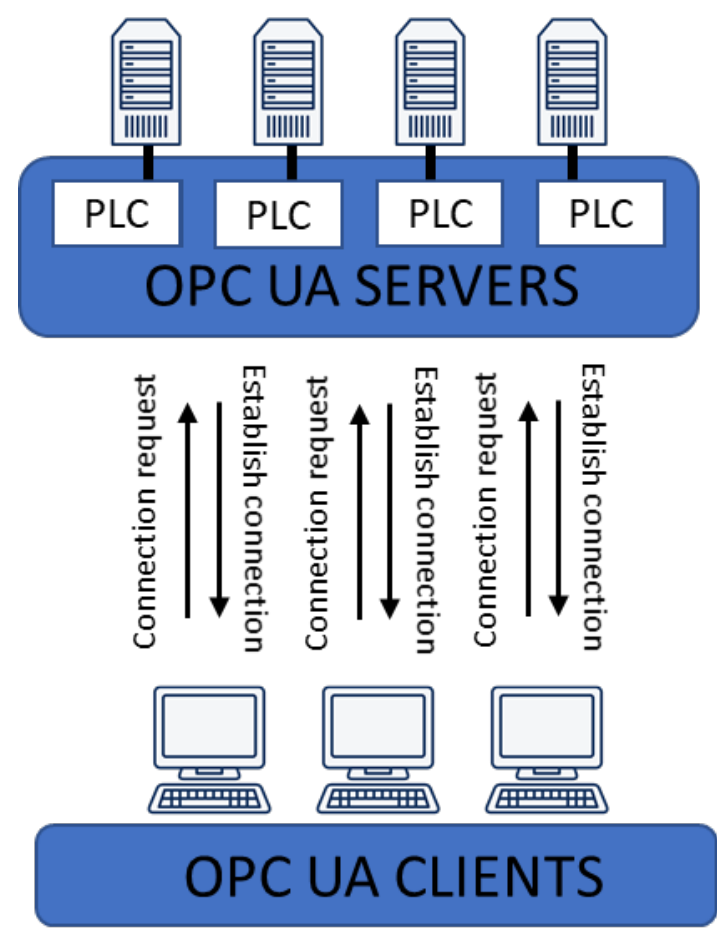

Figure 5. Schema of the OPC UA client/server connection.

This type of data exchange standard is a safe, reliable, manufacturer-independent, and platformindependent industrial communication. It enables secure data exchange between hardware platforms from different vendors and across operating systems. It is a way to extract data from PLCs and computers in order to analyse the productivity of production systems, optimize machine parameters, or plan predictive maintenance. 


\subsection{Modelling DT through machine states}

This section will describe how the DT of the I4.0 Lab was developed with Simulink. Noting that all the stations in the laboratory present the same features and similar components, the DT is developed for a single station and then replicated and tested for the other stations. As an example, Figure 6 shows the Front Cover Station, that will be taken as a reference to illustrate the DT development here proposed. The same procedure can be replicated for each station of the line, taking into consideration the characteristics of each machine of the production line itself.

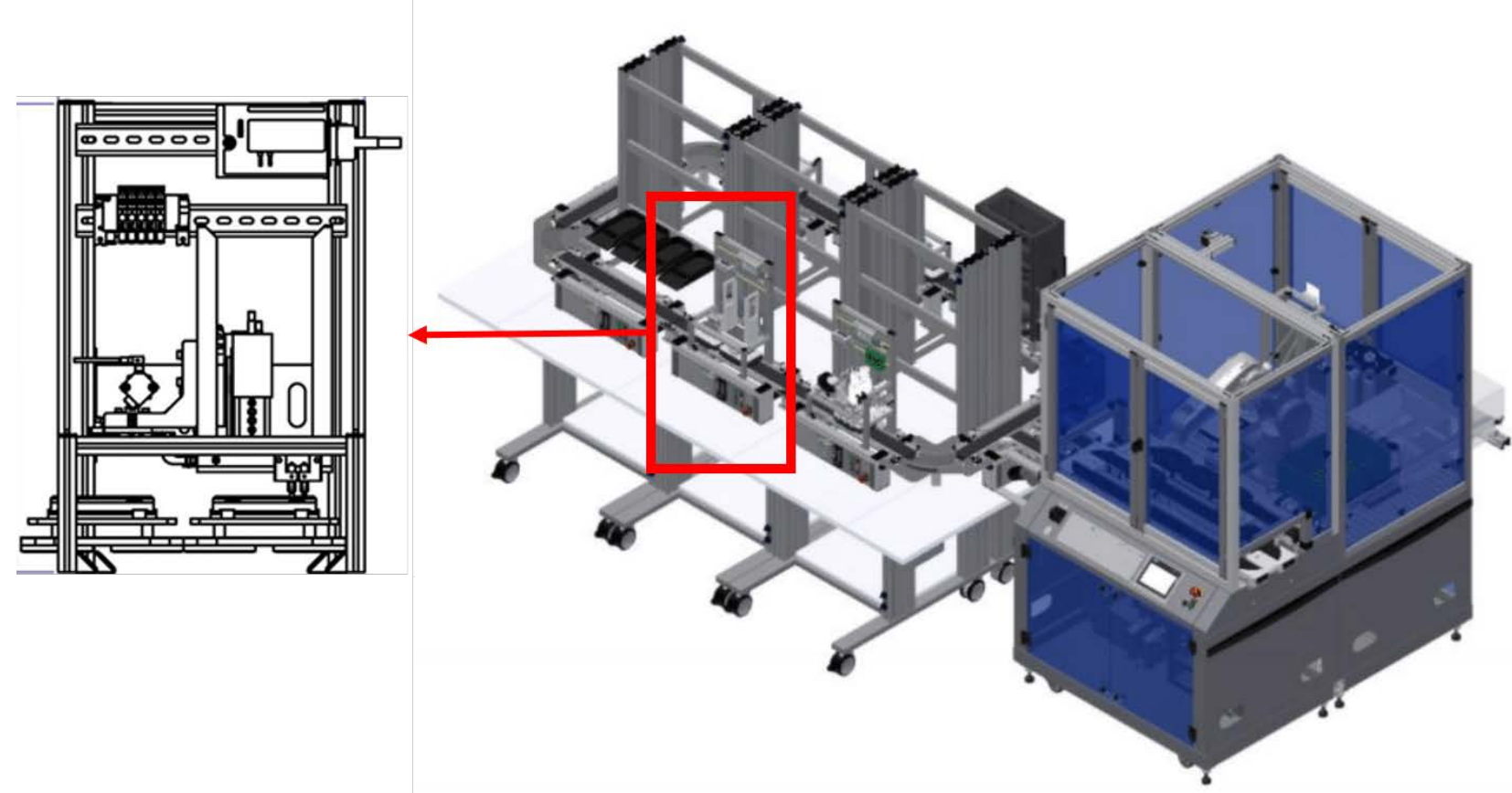

Figure 6. On the right the overall structure of the Industry 4.0 laboratory of the Politecnico di Milano, on the left the front cover station considered for the DT application (https://www.festodidactic.com/int-en/).

The DT development consists in building a virtual copy of each station in Simulink, that replicates its behaviour while the production is running, and connecting them, in a way that simulation is realtime synchronized with the field. As identified in the architecture of Figure 2, the two major identified steps are firstly to find a way to extract the data in real time and successively to find the right model to replicate the station inside the virtual environment. 
When it comes to extract a big set of data in real time on Simulink through OPC UA Toolbox with a simple MATLAB function, some tests in I4.0 Lab proved that this solution gives a big delay between the real values displayed in the HMI of the station and the ones on Simulink. The use of a simple MATLAB Function wastes time in the connection at each cycle during the simulation. For this reason, another MATLAB Function has been taken into account: The Level-2 MATLAB ${ }^{\circledR}$ Sfunction (https://it.mathworks.com/help/simulink/sfg/writing-level-2-matlab-s-functions.html). Thanks to this function it was possible to connect a client to the PLCs of the station only once at the start of the simulation. Then, thanks to the OPC UA toolbox, knowing the path of each needed signal, it was possible to reach and read each corresponding value at every sample time. The first step to construct the DT was the building of the mentioned Level-2 MATLAB ${ }^{\circledR}$ S-function to visualize the data from the field in Simulink. The functions Application Programming Interface (API) allows to use Matlab language to create custom blocks with multiple input and output ports, capable of handling any type of signal produced by a Simulink model. This function itself comprises a set of callback methods, that the Simulink engine invokes when updating or simulating the model. The callback methods perform the actual work of initializing and computing the outputs of the block defined by the S-function. Each block of Level-2 MATLAB ${ }^{\circledR}$ S-function used to acquire data use basically three methods:

- The Setup, where the number of Inputs and Outputs of the function is defined, such as their DataType (i.e. int, boolean, etc.), Complexity and Dimension;

- The Start, the most interesting functionality of the Matlab block. This method allows to do the connection to the server only at the start of the simulation of Simulink and to store in memory the addresses of the variables in the Level-2 Matlab S-Function block memory;

- The Output, that permits to associate each PLC signal address to a variable. When the output is set, the real-time values of the PLC signals became the Output of the Level-2 Matlab S- 
function block.

The production line must be replicated starting from these data extracted from Simulink. Being an advanced simulation, the DT needs to rely on a properly designed Data Model to construct it. Each machine can be modelled through a set of machine states identified in literature [80]. In alignment with the machine states found in literature, the identified states in the stations of the laboratory are [81]:

- Idle: the conveyor in the module is moving, but no operation is performed, the machine is waiting for a piece to work;

- Working: the machine is performing an operation;

- Energy-saving mode: the machine is on, but the belt is not moving to save energy when there is no piece to work immediately;

- Fault: the machine is down due to abnormal behaviour and shows an error message in the HMI. Only when the fault is fixed and the operator responds to the error message in the HMI, the work cycle resumes. The machine is in fault state in case the emergency button is triggered by the operator or there is a machine error.

Finally, Figure 7 on the left shows how the values acquired were combined to replicate in real-time the machine states of each station in laboratory. As it is showed in Figure 7 on the right, an output number was assigned for each machine state and that permits to univocally identify the machine state and to make analyses. 


\begin{tabular}{|ccccc|}
\hline $\begin{array}{c}\text { Belt } \\
\left(x Q A \_A 1\right)\end{array}$ & $\begin{array}{c}\text { working position } \\
\text { sensor } \\
(\text { xBG1) }\end{array}$ & Em_Button & Machine Error & Machine State \\
\hline 1 & 0 & 0 & 0 & Idle \\
\hline 1 & 1 & 0 & 0 & Idle \\
\hline 0 & 1 & 0 & 0 & Working Mode \\
\hline $0 / 1$ & $0 / 1$ & 1 & $0 / 1$ & $\begin{array}{c}\text { Emergency button } \\
\text { triggered }\end{array}$ \\
\hline $0 / 1$ & 1 & $0 / 1$ & 1 & Machine Error \\
\hline & Else & & & Energy-safe Mode \\
\hline
\end{tabular}

\begin{tabular}{|c|c|}
\hline Machine State & Output Number \\
\hline Idle & 1 \\
\hline Working Mode & 2 \\
\hline $\begin{array}{c}\text { Energy-safe Mode } \\
\text { Emergency button } \\
\text { triggered }\end{array}$ & 3 \\
\hline Machine Error & 4 \\
\hline
\end{tabular}

Figure 7. Machine States created by combining the signals from the PLC.

The DT model in Simulink is showed in Figure 9: from the left there are firstly the functions that extract the data - through the Level-2 MATLAB ${ }^{\circ}$ S-function - from the front cover station with the mentioned OPC UA protocol. Similar modelling has been elaborated also for the other stations of the line. In the middle of Figure 9, the three functions under the name "Machine state identification" identify the state of the machine analysing the acquired data. Finally, the functions for: energy consumption computation in real time (above), knowing machine state and the power consumption; data storage (below), to extract the data also outside the Matlab environment.

\subsection{Functionalities of the proposed Digital Twin}

Once identified the combination of sensors that gives the exact machine state in laboratory, it was possible to better represent a single station of the production line in a digital environment. The following functionalities of the DT were created:

- Energy consumption computation of the station;

- Collection of the data (sensors and actuators) read from the PLCs and their visualization in a graphical user interface (GUI) Figure 8;

- Tracking of each carrier inside the production line. 
Energy consumption is a topic that was already treated in literature based on the machine states of a single machine [80,82]. The function that computes the energy consumption, identified in Figure 9 as "Energy consumption computation" function, was created as an example to prove the multidisciplinarity of the Digital twin environment within the H2020 MAYA project as was discussed in [81]. It takes as Inputs the machine states, the instantaneous power from the Energy box server and the sample frequency of the running simulation. The function works performing these steps:

- It counts how long the station was in each machine state $i$ : $t_{i}$;

- It computes the mean value of the Power in each machine state $i$ during the simulation: $P_{i}$;

- It computes the Energy consumption for each machine state $i: E_{i}=P_{i}{ }^{*} t_{i}$;

Both the data collected and analysed can be visualised in the GUI represented in Figure 8.

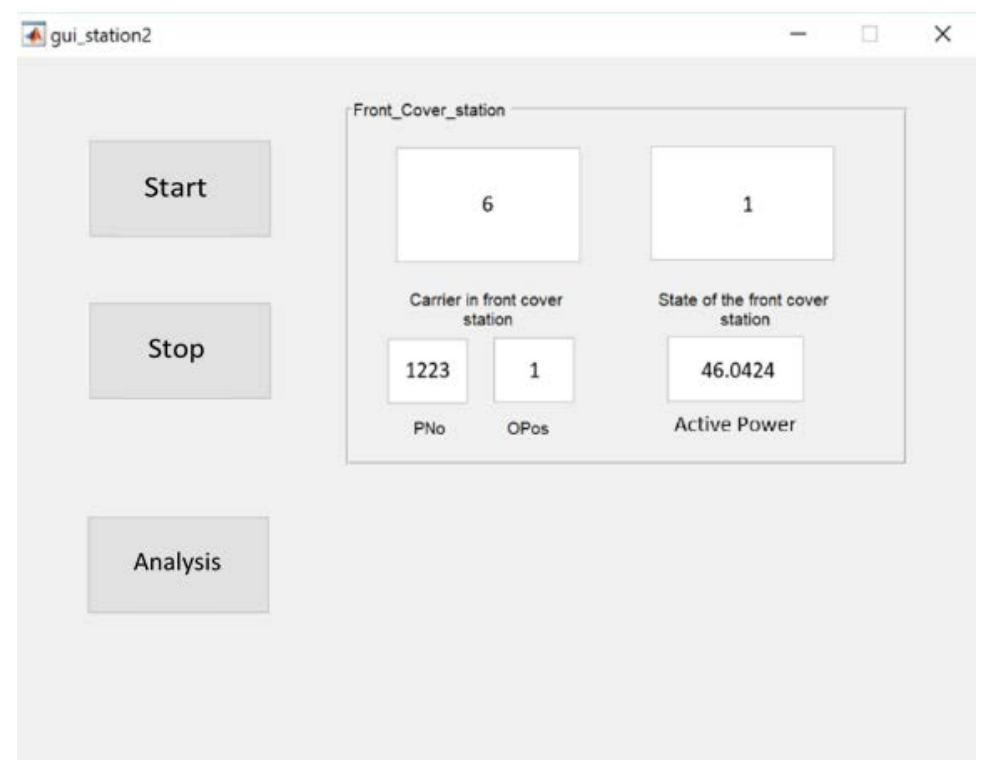

Figure 8. Graphical User Interface (GUI) in Matlab/Simulink to visualize the information of the front cover station in a user-friendly way.

This allows easy usage of the DT of Figure 9, since the user is not required to know the code that leads to the creation of the DT, the data extraction is clearer and more readable. All these data may be stored inside the MATLAB workspace and if the dataset is too big, the function "storage of 
useful data” of Figure 9 can be used to store data in a “.txt” file at each time step.

The last functionality of the DT is given by the usage of the stored data to track the production. From the "Analysis" button of the GUI in Figure 8, when the simulation has been stopped, the data can be analysed to understand the steps performed by the carrier during its passage through the station. This functionality was created to improve the traceability of the product in line with the H2020 PERFoRM project (http://www.horizon2020-perform.eu/). 


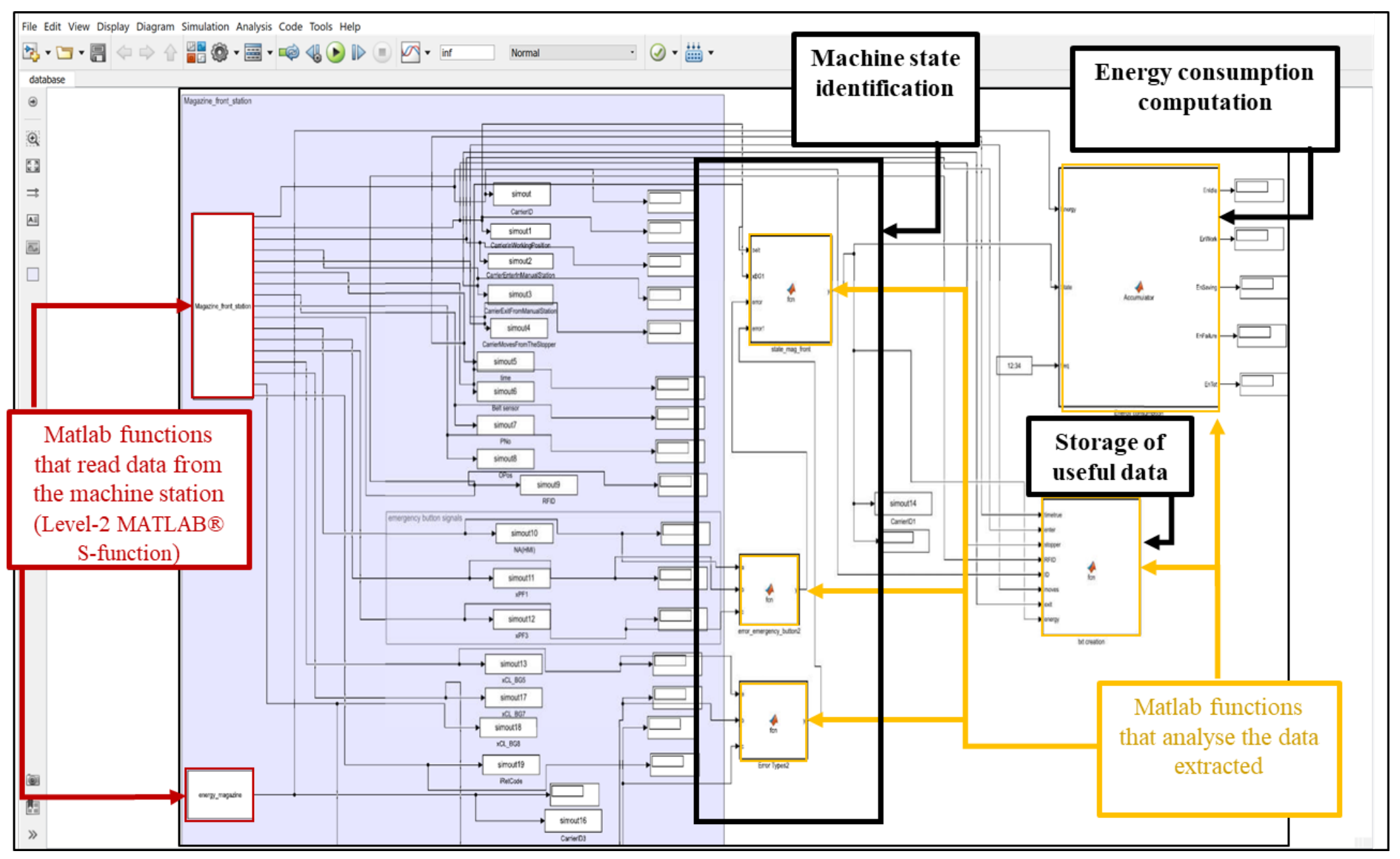

Figure 9. DT Simulink environment of the I4.0 Lab. 


\subsection{Results of the DT in laboratory}

The DT environment developed in the "DT creation" section, showed in Figure 9, was used to prove how a first introduction of the DT might bring benefits to the production and, in particular, to investigate the impact of connecting the DT to the MES system.

Taking into consideration the MES functions reported by the MESA [12], two of them clearly receive a positive impact through the addition of a DT in a production system (Figure 2) :

- $\quad$ Data collection and acquisition: From the data collected in real time to identify the machine states, it is also possible to obtain the energy consumption data, also in real time [83]. This application case gives the proof that with the DT environment the analysed data are stored and analysed in real-time, as in the MES. The important aspect is that all data from the field are stored in the DT as showed in Figure 10 (solid black line), increasing the number of collected data, avoiding sensors and actuators data loss. This represents a benefit, reminding that the MES system only stores aggregated data, after elaborations and analyses. Raw data from the field are lost Figure 10 (solid black line).

- $\quad$ Product tracking and genealogy: The importance of storing the acquired data from the field from sensors and actuators is underlined in the tracking of the piece. With more data, it is possible to obtain a higher level of granularity of the historical track of the product with respect to the tracking given by the MES system inside the laboratory Figure 10 (dotted black line). The data tracking by the MES system is only related to start and end of the operation Figure 10 (dotted black line), giving a picture in real time of the current situation of the 
completed and non-completed jobs. With the DT it is possible to save the history of the information from the field, retrieving - for example - where the carrier was in the system at a certain time in the past. This could not be done by the MES that provides the location of the carrier only "now" (i.e. at the current time).

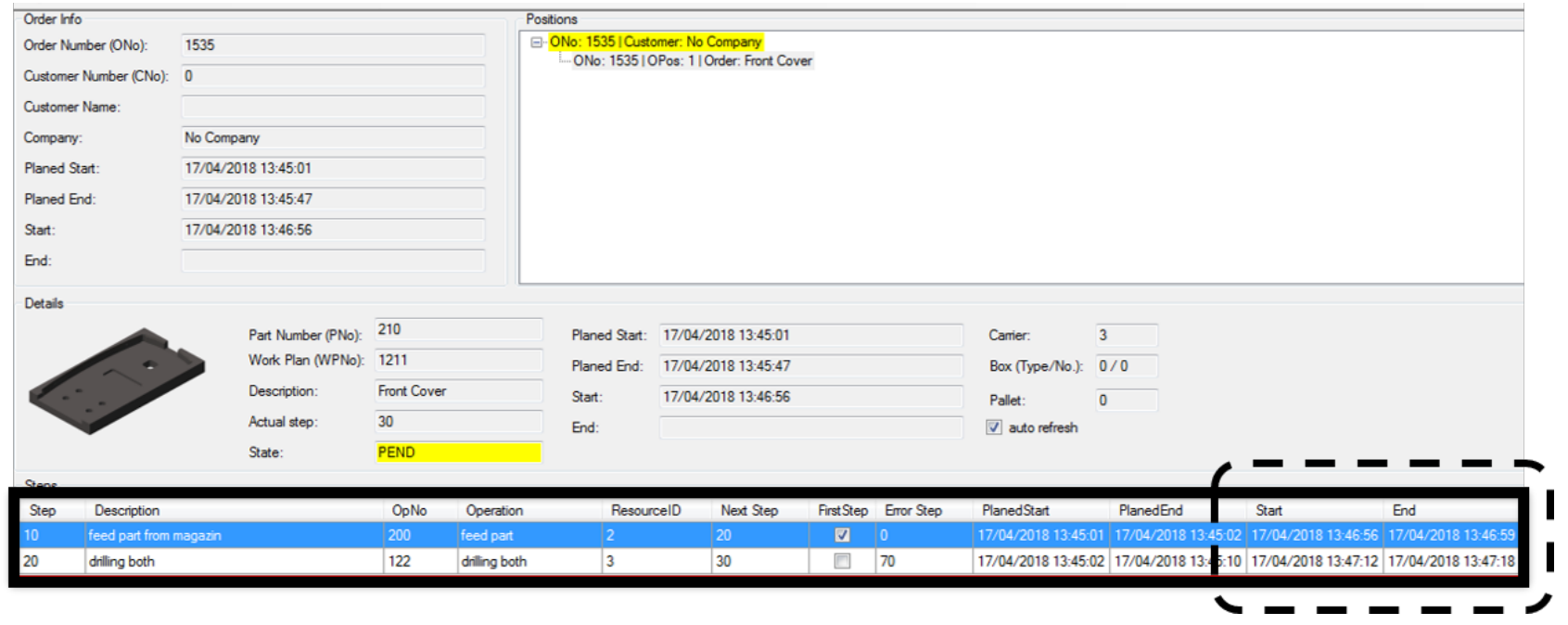

Figure 10. MES interface in laboratory.

Another improvement given by the proposed DT is the possibility to acquire data that come from different sources in the same environment, for example data from two different PLCs. To sum up, benefits are of a double nature:

- In real time data are collected in the digital environment can be read and analysed, stored inside or outside the Matlab virtual environment;

- With a historical data perspective, the stored more granular data can be used for a posteriori deeper analysis. 


\section{Discussion}

The proposed DT poses the basis to overcome the gaps from Section 3.4. The analysed DTs are limited in the number of services offered, never extended to the entire set of services of reference given by [21], and they are usually not integrated with the existent control system, making the DTs closer to a "Digital Shadow" [18] without reaching the full DT potentials mentioned in the "Introduction” section.

In the present state of research progress, the proposed DT has the following characteristics.

The environment created in laboratory is focused on the energy consumption computation, but the proposed architecture can be used to extract any data from the servers available in laboratory. The Simulink environment can be used to perform different analyses, also on the same dataset, with the same procedure showed for energy consumption. For this reason, it is a good DT environment not only for the services of "monitoring” and "energy consumption”, but also for the others as it can be seen in Table 1 . An exception must be done for the "Product virtual maintenance or operations" service, because there is not already a defined Matlab/Simulink tool that could transform the sensor data into a 3D guideline for the operator. There is instead the possibility to extend the GUI created into an HMI guide for the user through the collected data.

The available MES in laboratory is supplied with a TCP/IP communication language that allows to write messages to the MES itself, also from the Matlab/Simulink environment; it seems to be a good test bed to experimentally understand how the DT should be integrated inside a closed system already controlled by a MES. In fact, the laboratory MES is not completely open and programmable but is flexible enough to perform a first attempt of integration with the DT created in Simulink in future works. 
These two peculiarities of the presented DT application can be considered an improvement with respect to the others DT presented in Table 1 and set the research work in a focused direction, considering that:

1. In most of the cases, nothing is mentioned about the general potentiality of the created DT; they mostly show the single analysis done to create the DT without mentioning if it is possible to perform other analyses on it.

2. Only in one case the DT is constructed in Android application to be itself a MES for the system [43], in the other cases, already showed in Section 3, the integration with the MES is mentioned only in a theoretical way.

Summing up, this paper proposes a simulation model that is still in the realm of Digital Shadows but poses the basis to achieve a complete DT, that focuses on monitoring machine states and their relative energy consumption and that allows different analyses in the same environment related to the different services that can be offered from the DT [21], with the future possibility to integrate the DT with the MES of the laboratory in order to perform decision making from the DT, reaching the full DT potential as a properly DT defined by [18].

\section{Conclusions and future work}

In the most recent years, the DT in industry has received a relevant attention from both researchers and practitioners. Manufacturing is one of the most promising contexts where the DT may be successfully applied for concrete benefits in terms of maintenance and operations monitoring and optimization.

The article frames the research on DT as follows. Nowadays production systems are still "traditionally" controlled with the automation pyramid. However, the advent of CPS may lead to less hierarchical and more flexible control approaches. CPS are by nature cyber and physical, with a deep connection between the two worlds. In 
particular, the cyber part may host or be connected to simulations that are thus synchronized with the field (that literature commonly denominates DT). However, to have a full DT it is not only necessary to have data coming from the field to simulation, but also to be able to act from the simulation to the field, by offering monitoring and optimization services and by actuating decisions on the production control system [18]. For this reason, DT in their full functionalities may be included in new more flexible and flatter production control approaches, whose way is opened thanks to the CPS. This paper brings a contribution to the research on this topic, by firstly exploring the applications of the DT in manufacturing and the related services offered by them with reference to [21]. From this analysis, some misalignment was identified between the DT implementation and its description in literature:

o Each DT offers only some of the services that such platform should offer [21];

o Each DT takes data from the real systems, but this communication is never bidirectional with the field.

This paper also proposes the development of a DT of a laboratory line to lay the groundwork in overcome these gaps. The suggested DT monitors energy consumption during line operations based on machine states. The purposes of this development are the following:

The DT constructed in laboratory seems to be a good example of a single environment that offers the highest number of services promised by the introduction of the DT [21], from what appeared in literature until now;

$>$ The DT can give the basis to future works on the integration of the DT with the control system, that is generally a MES when the system is closed and proprietary.

The connection of the DT to the production system of the laboratory has brought to 
specific extensions of the functions of the MES of the laboratory production line, with respect to those mentioned by [12]. The acquisition of more data from the field guaranteed a more detailed information on the production with respect to the existent MES system that controls the line, demonstrating that the use of DT may enhance the availability of the field data, to be elaborated for a more consistent autonomous decision making, exploiting the presence of intelligent systems, such as CPS. This takes particular importance with respect to the Industry 4.0 wave, where data take the leading role to a higher comprehension of industrial processes and interactions for a deep digital transformation. Companies are at different levels of readiness and maturity in this process $[84,85]$ and this contribution aims at demonstrating practical implications of the use of DT for those companies willing to invest in the direction of the digital transformation.

Future works should close the control loop, by using the MES to act on the system, according to specific decision making and management rules. The developed DT proposed in this paper complements the MES of a closed proprietary system adding additional information, without sending any feedback to the MES. The open challenge is then to allow the DT to control the processes of a line also in closed proprietary systems, controlled by a MES system. It is extremely important to investigate this aspect to achieve more benefits from the DT, given that most of the production systems are ruled by a MES. In this way, decision making on important aspects, such as scheduling, energy consumption, safety, maintenance and quality management, could be made in a fully reactive way through DT to the MES. This is possible when the DT not only provides a richer information flow to the databases (as demonstrated by this contribution), but also with a proper structuring of rules that are provided to the MES. As emerged also from section 3, this is possible only thanks to the use of proper data 
models, to store data and on which rules would be written, such as reported by [19]. In other words, future work should leverage on the proposed work to elaborate a full DT [18].

\section{Acknowledgements}

This project has received funding from the European Union's Horizon 2020 research and innovation programmes under grant agreements No 678556 (correspondent to the project shortly entitled "MAYA", "MultidisciplinArY integrated simulAtion and forecasting tools, empowered by digital continuity and continuous real world synchronization, towards reduced time to production and optimization”) and No 680435 (correspondent to the project shortly entitled "PERFoRM", "Production harmonizEd Reconfiguration of Flexible Robots and Machinery”).

This research work has been possible thanks to the use of the Industry 4.0 Laboratory of the School of Management of the Politecnico di Milano (lab site: www.industry40lab.org).

\section{References}

[1] A. Kusiak, Smart Manufacturing, Int. J. Prod. Res. (2017).

[2] L. Monostori, Cyber-physical production systems: Roots from manufacturing science and technology, At-Automatisierungstechnik. (2015). doi:10.1515/auto2015-0066.

[3] J. Lee, B. Bagheri, H.A. Kao, A Cyber-Physical Systems architecture for Industry 4.0-based manufacturing systems, Manuf. Lett. 3 (2015) 18-23. doi:10.1016/j.mfglet.2014.12.001. 
[4] L. Cattaneo, L. Fumagalli, M. Macchi, E. Negri, Clarifying Data Analytics Concepts for Industrial Engineering, IFAC-PapersOnLine. 51 (2018) 820-825. doi:10.1016/j.ifacol.2018.08.440.

[5] V. Roblek, M. Meško, A. Krapež, A Complex View of Industry 4.0, SAGE Open. 6 (2016). doi:10.1177/2158244016653987.

[6] E. Negri, L. Fumagalli, M. Macchi, A Review of the Roles of Digital Twin in CPS-based Production Systems, Procedia Manuf. 11 (2017) 939-948. doi:10.1016/j.promfg.2017.07.198.

[7] E.M. Kraft, The US Air Force Digital Thread / Digital Twin - Life Cycle Integration and Use of Computational and Experimental Knowledge II . The Evolution of Integrated Computational / Experimental Fluid Dynamics, (2016) 1-22. doi:10.2514/6.2016-0897.

[8] ISO/IEC, ISO/IEC 62264-2, Enterprise-control system - Part 2: Objects and attributes for enterprise-control system integration, 2012.

[9] R. Govindaraju, K. Putra, A methodology for Manufacturing Execution Systems (MES) implementation, in: IOP Conf. Ser. Mater. Sci. Eng., 2016. doi:10.1088/1757-899X/114/1/012094.

[10] MESA INTERNATIONAL, MES Explained : A High Level Vision, White Pap. (1997) 1-25.

[11] D. Zuehlke, SmartFactory-Towards a factory-of-things, in: Annu. Rev. Control, 2010. doi:10.1016/j.arcontrol.2010.02.008.

[12] MESA, MES Explained: A High Level Vision - MESA White Paper Number 6, 
(1997).

http://www.cpdee.ufmg.br/ seixas/PaginaII/Download/DownloadFiles/pap6.pdf.

[13] B.S. De Ugarte, A. Artiba, R. Pellerin, Manufacturing execution system - A literature review, Prod. Plan. Control. 20 (2009) 525-539.

doi:10.1080/09537280902938613.

[14] R. Rosen, G. Von Wichert, G. Lo, K.D. Bettenhausen, About the importance of autonomy and digital twins for the future of manufacturing, IFAC-PapersOnLine. 28 (2015) 567-572. doi:10.1016/j.ifacol.2015.06.141.

[15] S. Weyer, M. Schmitt, M. Ohmer, D. Gorecky, Towards Industry 4.0 Standardization as the crucial challenge for highly modular, multi-vendor production systems, IFAC-PapersOnLine. 48 (2015) 579-584. doi:10.1016/j.ifacol.2015.06.143.

[16] S. Iarovyi, W.M. Mohammed, A. Lobov, B.R. Ferrer, J.L.M. Lastra, CyberPhysical Systems for Open-Knowledge-Driven Manufacturing Execution Systems, Proc. IEEE. 104 (2016) 1142-1154.

doi:10.1109/JPROC.2015.2509498.

[17] M. Garetti, P. Rosa, S. Terzi, Computers in Industry Life Cycle Simulation for the design of Product - Service Systems, Comput. Ind. 63 (2012) 361-369. doi:10.1016/j.compind.2012.02.007.

[18] W. Kritzinger, M. Karner, G. Traar, J. Henjes, W. Sihn, Digital Twin in manufacturing: A categorical literature review and classification, IFACPapersOnLine. 51 (2018) 1016-1022. doi:10.1016/j.ifacol.2018.08.474. 
[19] M. Garetti, L. Fumagalli, E. Negri, Role of ontologies for cps implementation in manufacturing, Manag. Prod. Eng. Rev. (2015). doi:10.1515/mper-2015-0033.

[20] E. Negri, L. Fumagalli, M. Garetti, L. Tanca, Requirements and languages for the semantic representation of manufacturing systems, Comput. Ind. 81 (2016) 5566. doi:10.1016/j.compind.2015.10.009.

[21] F. Tao, J. Cheng, Q. Qi, M. Zhang, H. Zhang, F. Sui, Digital twin-driven product design, manufacturing and service with big data, Int. J. Adv. Manuf. Technol. 94 (2018) 3563-3576. doi:10.1007/s00170-017-0233-1.

[22] M. Macchi, I. Roda, E. Negri, L. Fumagalli, Exploring the role of Digital Twin for Asset Lifecycle Management, IFAC-PapersOnLine. 51 (2018) 790-795. doi:10.1016/j.ifacol.2018.08.415.

[23] G. Avventuroso, M. Silvestri, P. Pedrazzoli, A Networked Production System to Implement Virtual Enterprise and Product Lifecycle Information Loops, IFACPapersOnLine. 50 (2017) 7964-7969. doi:10.1016/j.ifacol.2017.08.902.

[24] Q. Liu, H. Zhang, J. Leng, X. Chen, Digital twin-driven rapid individualised designing of automated flow-shop manufacturing system, Int. J. Prod. Res. 7543 (2018) 1-17. doi:10.1080/00207543.2018.1471243.

[25] D. Olivotti, S. Dreyer, B. Lebek, M.H. Breitner, Creating the foundation for digital twins in the manufacturing industry: an integrated installed base management system, Inf. Syst. E-Bus. Manag. 17 (2019) 89-116. doi:10.1007/s10257-018-0376-0.

[26] V. Souza, R. Cruz, W. Silva, S. Lins, V. Lucena, A Digital Twin Architecture 
Based on the Industrial Internet of Things Technologies, 2019 IEEE Int. Conf. Consum. Electron. ICCE 2019. (2019) 1-2. doi:10.1109/ICCE.2019.8662081.

[27] A. Angrish, B. Starly, Y.S. Lee, P.H. Cohen, A flexible data schema and system architecture for the virtualization of manufacturing machines (VMM), J. Manuf. Syst. 45 (2017) 236-247. doi:10.1016/j.jmsy.2017.10.003.

[28] R. Beregi, Á. Szaller, B. Kádár, Synergy of multi-modelling for process control, IFAC-PapersOnLine. 51 (2018) 1023-1028. doi:10.1016/j.ifacol.2018.08.473.

[29] Z. Kemény, R.J. Beregi, G. Erdos, J. Nacsa, The MTA SZTAKI Smart Factory: Platform for Research and Project-oriented Skill Development in Higher Education, in: Procedia CIRP, 2016: pp. 53-58. doi:10.1016/j.procir.2016.05.060.

[30] D. Botkina, M. Hedlind, B. Olsson, J. Henser, T. Lundholm, Digital Twin of a Cutting Tool, Procedia CIRP. (2018). doi:10.1016/j.procir.2018.03.178.

[31] Y. Cai, B. Starly, P. Cohen, Y.S. Lee, Sensor Data and Information Fusion to Construct Digital-twins Virtual Machine Tools for Cyber-physical Manufacturing, Procedia Manuf. 10 (2017) 1031-1042. doi:10.1016/j.promfg.2017.07.094.

[32] T. DebRoy, W. Zhang, J. Turner, S.S. Babu, Building digital twins of 3D printing machines, Scr. Mater. 135 (2017) 119-124. doi:10.1016/j.scriptamat.2016.12.005.

[33] F. Guo, F. Zou, J. Liu, Z. Wang, Working mode in aircraft manufacturing based on digital coordination model, Int. J. Adv. Manuf. Technol. 98 (2018) 1547- 
1571. doi:10.1007/s00170-018-2048-0.

[34] S. Haag, R. Anderl, Digital twin - Proof of concept, Manuf. Lett. 15 (2018) 6466. doi:10.1016/j.mfglet.2018.02.006.

[35] J. Liu, H. Zhou, G. Tian, X. Liu, X. Jing, Digital twin-based process reuse and evaluation approach for smart process planning, Int. J. Adv. Manuf. Technol. (2019). doi:10.1007/s00170-018-2748-5.

[36] P. Janda, Mechatronic Concept of Heavy Machine Tools, (2018) 0645-0652. doi:10.2507/29th.daaam.proceedings.093.

[37] N. Karanjkar, A. Joglekar, S. Mohanty, V. Prabhu, D. Raghunath, R. Sundaresan, Digital twin for energy optimization in an SMT-PCB assembly line, Proc. - 2018 IEEE Int. Conf. Internet Things Intell. Syst. IOTAIS 2018. (2019) 85-89. doi:10.1109/IOTAIS.2018.8600830.

[38] G.L. Knapp, T. Mukherjee, J.S. Zuback, H.L. Wei, T.A. Palmer, A. De, T. Debroy, Acta Materialia Building blocks for a digital twin of additive manufacturing, 135 (2017) 390-399. doi:10.1016/j.actamat.2017.06.039.

[39] A. Padovano, F. Longo, L. Nicoletti, G. Mirabelli, A Digital Twin based Service Oriented Application for a 4.0 Knowledge Navigation in the Smart Factory, IFAC-PapersOnLine. 51 (2018) 631-636. doi:10.1016/j.ifacol.2018.08.389.

[40] K.T. Park, S.J. Im, Y.S. Kang, S. Do Noh, Y.T. Kang, S.G. Yang, Serviceoriented platform for smart operation of dyeing and finishing industry, Int. J. Comput. Integr. Manuf. 32 (2019) 307-326.

doi:10.1080/0951192X.2019.1572225. 
[41] E. Sujová, H. Čierna, I. Zabińska, Application of digitization procedures of production in practice, Manag. Syst. Prod. Eng. 27 (2019) 23-28. doi:10.1515/mspe-2019-0004.

[42] J. Um, J. Popper, M. Ruskowski, Modular augmented reality platform for smart operator in production environment, Proc. - 2018 IEEE Ind. Cyber-Physical Syst. ICPS 2018. (2018) 720-725. doi:10.1109/ICPHYS.2018.8390796.

[43] P.D. Urbina Coronado, R. Lynn, W. Louhichi, M. Parto, E. Wescoat, T. Kurfess, Part data integration in the Shop Floor Digital Twin: Mobile and cloud technologies to enable a manufacturing execution system, J. Manuf. Syst. 48 (2018) 25-33. doi:10.1016/j.jmsy.2018.02.002.

[44] J. Vachalek, L. Bartalsky, O. Rovny, D. Sismisova, M. Morhac, M. Loksik, The digital twin of an industrial production line within the industry 4.0 concept, in: Proc. 2017 21st Int. Conf. Process Control. PC 2017, 2017. doi:10.1109/PC.2017.7976223.

[45] C.C. Wei, Y.T. Lee, K.S. Cao, W.C. Lee, Implementation of a data acquisition system for heterogeneous machines, SII 2017 - 2017 IEEE/SICE Int. Symp. Syst. Integr. 2018-Janua (2018) 232-235. doi:10.1109/SII.2017.8279217.

[46] H. Zhang, Q. Liu, X. Chen, D. Zhang, J. Leng, A Digital Twin-Based Approach for Designing and Multi-Objective Optimization of Hollow Glass Production Line, IEEE Access. 5 (2017) 26901-26911. doi:10.1109/ACCESS.2017.2766453.

[47] S. Konstantinov, M. Ahmad, K. Ananthanarayan, R. Harrison, The Cyberphysical E-machine Manufacturing System: Virtual Engineering for Complete 
Lifecycle Support, in: Procedia CIRP, The Author(s), 2017: pp. 119-124. doi:10.1016/j.procir.2017.02.035.

[48] X.V. Wang, L. Wang, Digital twin-based WEEE recycling, recovery and remanufacturing in the background of Industry 4.0, Int. J. Prod. Res. 0 (2018) 111. doi:10.1080/00207543.2018.1497819.

[49] J. David, A. Lobov, M. Lanz, Leveraging Digital Twins for Assisted Learning of Flexible Manufacturing Systems, Proc. - IEEE 16th Int. Conf. Ind. Informatics, INDIN 2018. 589 (2018) 529-535. doi:10.1109/INDIN.2018.8472083.

[50] V. Toivonen, M. Lanz, H. Nylund, H. Nieminen, The FMS Training Center - A versatile learning environment for engineering education, in: Procedia Manuf., Elsevier B.V., 2018: pp. 135-140. doi:10.1016/j.promfg.2018.04.006.

[51] J. Um, S. Weyer, F. Quint, Plug-and-Simulate within Modular Assembly Line enabled by Digital Twins and the use of AutomationML, IFAC-PapersOnLine. 50 (2017) 15904-15909. doi:10.1016/j.ifacol.2017.08.2360.

[52] F. Yao, A. Keller, M. Ahmad, B. Ahmad, R. Harrison, A.W. Colombo, Optimizing the Scheduling of Autonomous Guided Vehicle in a Manufacturing Process, Proc. - IEEE 16th Int. Conf. Ind. Informatics, INDIN 2018. (2018) 264269. doi:10.1109/INDIN.2018.8471979.

[53] M. Ayani, M. Ganebäck, A.H.C. Ng, Digital Twin: Applying emulation for machine reconditioning, in: Procedia CIRP, Elsevier B.V., 2018: pp. 243-248. doi:10.1016/j.procir.2018.03.139.

[54] A. da S. Barbosa, F.P. Silva, L.R.D.S. Crestani, R.B. Otto, Virtual assistant to 
real time training on industrial environment, Adv. Transdiscipl. Eng. 7 (2018) 33-42. doi:10.3233/978-1-61499-898-3-33.

[55] H. Brandtstaedter, C. Ludwig, L. Hubner, E. Tsouchnika, A. Jungiewicz, U. Wever, Digital Twins for Large Electric Drive Trains, Pet. Chem. Ind. Conf. Eur. Conf. Proceedings, PCIC Eur. 2018-June (2018). doi:10.23919/PCICEurope.2018.8491413.

[56] F. Longo, L. Nicoletti, A. Padovano, Ubiquitous knowledge empowers the Smart Factory: The impacts of a Service-oriented Digital Twin on enterprises’ performance, Annu. Rev. Control. (2019). doi:10.1016/j.arcontrol.2019.01.001.

[57] W. Luo, T. Hu, W. Zhu, F. Tao, Digital twin for CNC machine tool, (2018) 2-5. doi:10.1007/s12652-018-0946-5.

[58] K.T. Park, Y.W. Nam, H.S. Lee, S.J. Im, S. Do Noh, J.Y. Son, H. Kim, Design and implementation of a digital twin application for a connected micro smart factory, Int. J. Comput. Integr. Manuf. 00 (2019) 1-19. doi:10.1080/0951192X.2019.1599439.

[59] S. Rabah, A. Assila, E. Khouri, F. Maier, F. Ababsa, V. Bourny, P. Maier, F. Mérienne, Towards improving the future of manufacturing through digital twin and augmented reality technologies, Procedia Manuf. 17 (2018) 460-467. doi:10.1016/j.promfg.2018.10.070.

[60] A. Ardanza, A. Moreno, Á. Segura, M. de la Cruz, D. Aguinaga, Sustainable and flexible industrial human machine interfaces to support adaptable applications in the Industry 4.0 paradigm, Int. J. Prod. Res. 7543 (2019) 0-15. doi:10.1080/00207543.2019.1572932. 
[61] V. Kuts, G.E. Modoni, W. Terkaj, T. Tähemaa, M. Sacco, T. Otto, Exploiting Factory Telemetry to Support Virtual Reality Simulation in Robotics Cell, in: L.T. De Paolis, P. Bourdot, A. Mongelli (Eds.), Augment. Reality, Virtual Reality, Comput. Graph. Fourth Int. Conf. Part I, 2017. doi:10.1007/978-3-31940621-3.

[62] E. Frontoni, J. Loncarski, R. Pierdicca, M. Bernardini, M. Sasso, Cyber Physical Systems for Industry 4.0: Towards Real Time Virtual Reality in Smart Manufacturing, in: L.T. De Paolis, P. Bourdot (Eds.), Correct. to Augment. Reality, Virtual Reality, Comput. Graph., 2018: pp. E1-E1. doi:10.1007/978-3319-95282-6_51.

[63] J.O. Oyekan, W. Hutabarat, A. Tiwari, R. Grech, M.H. Aung, M.P. Mariani, L. López-Dávalos, T. Ricaud, S. Singh, C. Dupuis, The effectiveness of virtual environments in developing collaborative strategies between industrial robots and humans, Robot. Comput. Integr. Manuf. 55 (2019) 41-54. doi:10.1016/j.rcim.2018.07.006.

[64] J. Spranger, R. Buzatoiu, A. Polydoros, L. Nalpantidis, E. Boukas, Humanmachine interface for remote training of robot tasks., IST 2018 - IEEE Int. Conf. Imaging Syst. Tech. Proc. (2018) 1-5. doi:10.1109/IST.2018.8577081.

[65] J. Xie, X. Wang, Z. Yang, S. Hao, Virtual monitoring method for hydraulic supports based on digital twin theory, Min. Technol. Trans. Inst. Min. Metall. 128 (2019) 77-87. doi:10.1080/25726668.2019.1569367.

[66] S. Zambal, C. Eitzinger, M. Clarke, J. Klintworth, P.Y. Mechin, A digital twin for composite parts manufacturing : EEffects of defects analysis based on 
manufacturing data, in: Proc. - IEEE 16th Int. Conf. Ind. Informatics, INDIN 2018, IEEE, 2018: pp. 803-808. doi:10.1109/INDIN.2018.8472014.

[67] P. Zheng, T.J. Lin, C.H. Chen, X. Xu, A systematic design approach for service innovation of smart product-service systems, J. Clean. Prod. 201 (2018) 657-667. doi:10.1016/j.jclepro.2018.08.101.

[68] S. Anand, O. Ghalsasi, B. Zhang, A. Goel, S. Reddy, S. Joshi, G. Morris, Additive manufacturing simulation tools in education, 2018 World Eng. Educ. Forum - Glob. Eng. Deans Counc. WEEF-GEDC 2018. (2019) 1-6. doi:10.1109/WEEF-GEDC.2018.8629689.

[69] F. Caputo, A. Greco, M. Fera, R. Macchiaroli, Digital twins to enhance the integration of ergonomics in the workplace design, Int. J. Ind. Ergon. 71 (2019) 20-31. doi:10.1016/j.ergon.2019.02.001.

[70] D. Dupláková, M. Flimel, J. Duplák, M. Hatala, S. Radchenko, F. Botko, Ergonomic rationalization of lighting in the working environment. Part I.: Proposal of rationalization algorithm for lighting redesign, Int. J. Ind. Ergon. 71 (2019) 92-102. doi:10.1016/j.ergon.2019.02.012.

[71] E.A. Loeken, A. Trulsen, A.M. Holsaeter, E. Wiktorski, D. Sui, R. Ewald, Design Principles Behind the Construction of an Autonomous Laboratory-Scale Drilling Rig, IFAC-PapersOnLine. 51 (2018) 62-69. doi:10.1016/j.ifacol.2018.06.356.

[72] A. Moreno, G. Velez, A. Ardanza, I. Barandiaran, Á.R. de Infante, R. Chopitea, Virtualisation process of a sheet metal punching machine within the Industry 4.0 vision, Int. J. Interact. Des. Manuf. 11 (2017) 365-373. doi:10.1007/s12008-016- 
0319-2.

[73] S. Sierla, V. Kyrki, P. Aarnio, V. Vyatkin, Automatic assembly planning based on digital product descriptions, Comput. Ind. 97 (2018) 34-46. doi:10.1016/j.compind.2018.01.013.

[74] L. Hu, N.T. Nguyen, W. Tao, M.C. Leu, X.F. Liu, M.R. Shahriar, S.M.N. Al Sunny, Modeling of Cloud-Based Digital Twins for Smart Manufacturing with MT Connect, in: Procedia Manuf., Elsevier B.V., 2018: pp. 1193-1203. doi:10.1016/j.promfg.2018.07.155.

[75] M.R. Shahriar, S.M.N. Al Sunny, X. Liu, M.C. Leu, L. Hu, N.T. Nguyen, MTComm based virtualization and integration of physical machine operations with digital-twins in cyber-physical manufacturing cloud, in: Proc. - 5th IEEE Int. Conf. Cyber Secur. Cloud Comput. 4th IEEE Int. Conf. Edge Comput. Scalable Cloud, CSCloud/EdgeCom 2018, IEEE, 2018: pp. 46-51. doi:10.1109/CSCloud/EdgeCom.2018.00018.

[76] X. Wang, Y. Guo, Y. Wang, Automatic detection of regions of interest in breast ultrasound images based on local phase information, Biomed. Mater. Eng. 26 (2015) S1265-S1273. doi:10.3233/BME-151424.

[77] L. Fumagalli, M. Macchi, A. Pozzetti, M. Taisch, G. Tavola, S. Terzi, New methodology for smart manufacturing research and education: The lab approach, Proc. Summer Sch. Fr. Turco. 13-15-Sept (2016) 42-47. https://www.scopus.com/inward/record.uri?eid=2-s2.085006043680\&partnerID=40\&md5=cdf822a3149be33e775b070189bad19d.

[78] M. Stopper, B. Katalinic, Service-oriented Architecture Design Aspects of OPC 
UA for Industrial Applications, Int. MultiConference Eng. Comput. Sci. II (2009) 18-21. doi:10.1.1.148.5549.

[79] L. Fumagalli, A. Polenghi, E. Negri, I. Roda, Framework for simulation software selection, J. Simul. (2019) 1-18. doi:10.1080/17477778.2019.1598782.

[80] M. Taisch, B. Stahl, F. Vaccari, A. Cataldo, A Production-State Based Approach for Energy Flow Simulation in Manufacturing Systems, in: 2013: pp. 227-234. doi:10.1007/978-3-642-41266-0_28.

[81] E. Negri, L. Fumagalli, C. Cimino, M. Macchi, FMU-supported simulation for CPS Digital Twin, Procedia Manuf. 28 (2019) 201-206. doi:10.1016/j.promfg.2018.12.033.

[82] S. Mousavi, S. Thiede, W. Li, S. Kara, C. Herrmann, An integrated approach for improving energy efficiency of manufacturing process chains, Int. J. Sustain. Eng. 9 (2016) 11-24. doi:10.1080/19397038.2014.1001470.

[83] E. Negri, L. Fumagalli, C. Cimino, M. Macchi, FMU-supported simulation for CPS Digital Twin, Procedia Manuf. CARV Int. Conf. Chang. Agil. Reconfigurable Virtual Prod. Nantes, 8th - 10th Oct. 2018. 00 (2018).

[84] A. De Carolis, M. Macchi, E. Negri, S. Terzi, Guiding manufacturing companies towards digitalization, 23rd ICE/IEEE Int. Technol. Manag. Conf. (2017) 503512. doi:10.1109/ICE.2017.8279925.

[85] A. De Carolis, M. Macchi, E. Negri, S. Terzi, A Maturity Model for Assessing the Digital Readiness of Manufacturing Companies, in: APMS 2017, Part I, IFIP AICT 513, 2017: pp. 13-20. doi:10.1007/978-3-319-66923-6. 
